\title{
Design of a Decoupled AP1000 Reactor Core Control System using Digital Proportional-Integral-Derivative (PID) Control Based on a Quasi-Diagonal Recurrent Neural Network (QDRNN)
}

Wei, Xinyu ${ }^{1}$ Wang, Pengfei, Zhao, Fuyu

School of Nuclear Science and Technology, Xi'an Jiaotong University, Xi'an, China

\begin{abstract}
The control system of the AP1000 reactor core uses the mechanical shim (MSHIM) strategy, which includes a power control subsystem and an axial power distribution control subsystem. To address the strong coupling between the two subsystems, an interlock between the two subsystems is used, which can only alleviate but not eliminate the coupling. Therefore, sometimes the axial offset (A0) cannot be controlled tightly, and the flexibility of load-following operation is limited. Thus, the decoupling of the original AP1000 reactor core control system is the focus of this paper. First, a two-node disperse dynamic model is established for the AP1000 reactor core to use PID control. Then, a digital PID control system based on a quasidiagonal recurrent neural network (QDRNN) is designed to decouple the original system. Finally, the decoupling of the control system is verified by the step signal and load-following condition. The results show that the designed control system can decouple the original system as expected and the AO can be controlled much more tightly. Moreover, the flexibility of the load following is increased.
\end{abstract}

Key words: AP1000 Reactor Core, Decouple, Disperse dynamic model, Quasi-diagonal recurrent neural network (QDRNN)

\footnotetext{
${ }^{1}$ Corresponding author xyuwei@xjtu.edu.cn
} 


\section{Introduction}

As the first Generation III+ reactor, the AP1000 is a two-loop pressurized water reactor (PWR) with $3400 \mathrm{MWth}$ power developed by Westinghouse that uses the forces of nature and simplicity of design to enhance plant safety and reduce construction costs (Schulz, 2006). Compared to the conventional Westinghouse 3-loop plant, the AP1000 adopts a different core control strategy, named mechanical shim (MSHIM). The MSHIM control strategy is basically characterized by the use of two separate digital rod control systems that automatically adjust the global core power and axial power distribution without soluble boron adjustment (Westinghouse Electric Company, 2011; Drudy et al., 2013).

Depending on its advancement, the MSHIM strategy has been employed by AP600, AP1000 and System 80+ reactors (Onoue et al., 2003), and the application of the mode to the IRIS reactor has been studied by Franceschini et al. (Franceschini and Petrovic, 2008). The MSHIM control system contains two subsystems, one the global power control subsystem that controls the core reactivity or temperature by a set of gray and black rods named M-banks and the other the axial offset (AO) control subsystem that controls the axial power distribution by a set of black rods named AO-banks. There are a total of 69 control assemblies in the AP1000: the M-banks are marked as MA, MB, MC, MD, M1, and $\mathrm{M} 2$, the AO-bank is marked as AO, and the shut-down banks are marked as SD1, SD2, D3, and SD4. The control rods are grouped as in Table 1 (Westinghouse Electric Company, 2010; Fetterman, 2009).

Table 1 List of AP1000 Control Rods 


\begin{tabular}{cccc}
\hline Group & Function & Type & Quantity \\
\hline MA & Power level control & Gray & 4 \\
MB & Power level control & Gray & 4 \\
MC & Power level control & Gray & 4 \\
MD & Power level control & Gray & 4 \\
M1 & Power level control & Black & 4 \\
M2 & Power level control & Black & 8 \\
AO & Axial power distribution & Black & 9 \\
SD1 & Shut-down & Black & 8 \\
SD2 & Shut-down & Black & 8 \\
SD3 & Shut-down & Black & 8 \\
SD4 & Shut-down & Black & 8 \\
\hline
\end{tabular}

The arrangement of the control rods in the AP1000 reactor core is shown in Fig. 1. $\begin{array}{llllllllllllllll}R & P & N & M & L & K & J & H & G & F & E & D & C & B & A\end{array}$

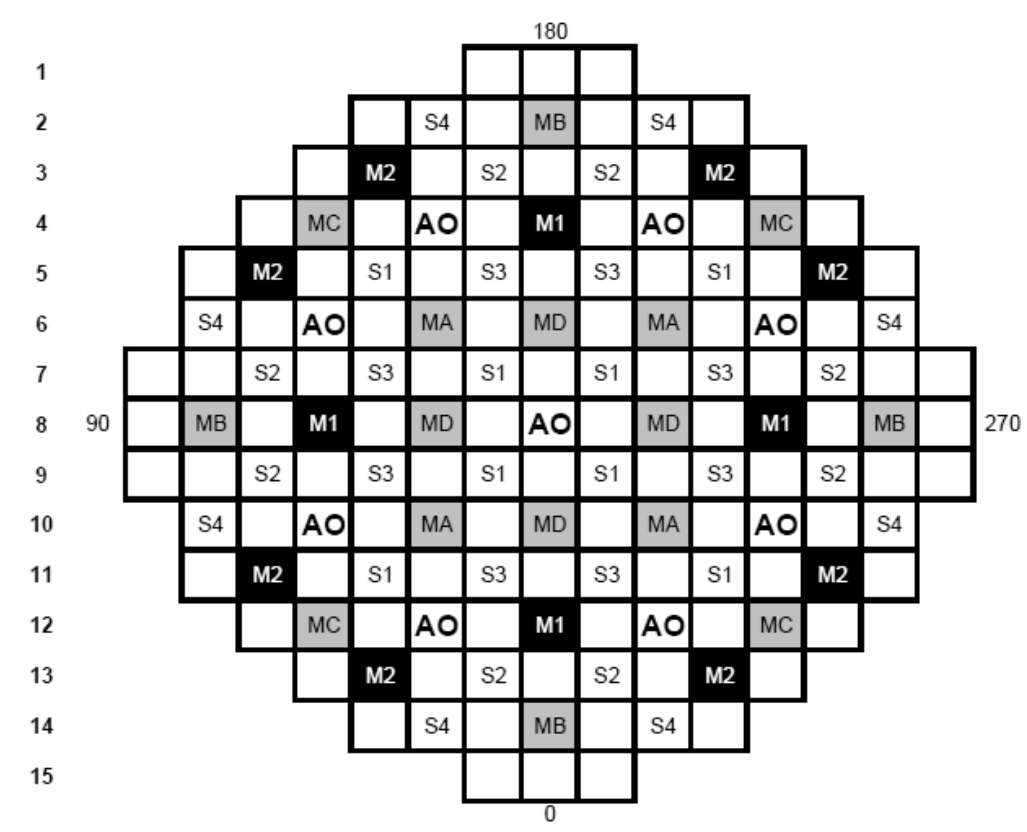

Fig. 1 Arrangement of the control rods assemblies in the AP1000 reactor core. MA, MB, MC, MD, $\mathrm{M} 1$, and M2 are M-banks, $\mathrm{AO}$ is AO-bank, SD1, SD2, D3, and SD4 are shut-down banks.

The M-banks consist of six control banks moving with a fixed overlap. The overlap scheme provides both positive and negative reactivity insertion capabilities in the event of an operational transient and can compensate for fuel depletion through the slow withdrawal 
of the M-banks during base load operation. To minimize the impact on the AO, MA, MB, MC, and MD are gray rods, M1 and M2 are black rods, and the overlap lengths are all 33\% of the rod height between neighboring banks. Due to its reasonable impact on the AO with limited motion, the AO-bank is a relatively high-worth bank rod and is limited to adjustment in a fixed region within the top half of the core. The overlap of the M-banks and the limitation of the AO-bank are shown in Fig. 2.

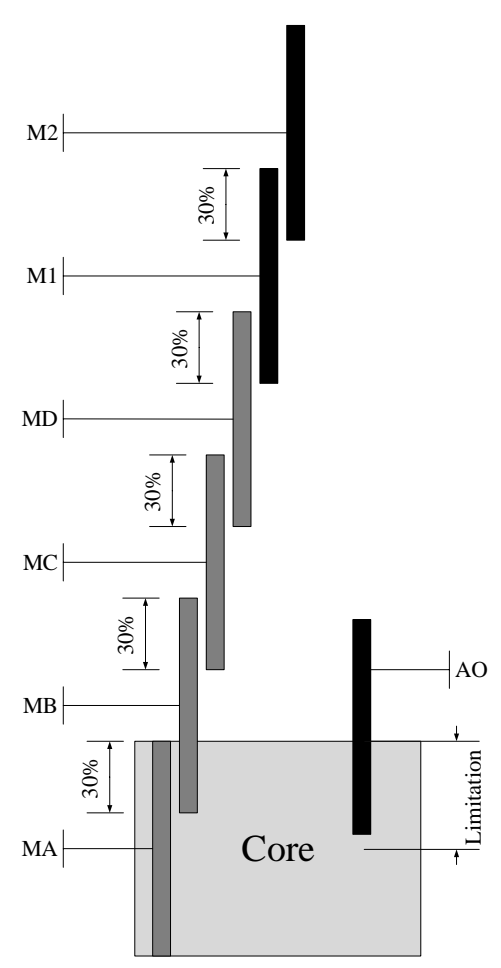

Fig. 2 Schematic of the overlap of M-banks and the movement limitation of the AO-bank.

Compared with the traditional operation mode in current-generation plants, the MSHIM employed by the AP1000 has many benefits. (1) MSHIM replaces the frequent manipulation of the soluble boron concentration during load-following with control rod movements, thereby avoiding excessive waste water generation during load-following and greatly simplifying the design of the chemical volume and control system. (2) The ability to load-follow can reach $95 \%$ of the cycle, while the ability of the current-generation plants 
can only reach $80 \%$ or even less (Westinghouse Electric Company, 2011; Zheng et al., 2010). (3) The control banks moved for power level control by the $M$ banks and axial power shape control by the AO bank are independent of each other and automatically controlled by the rod control system above $15 \%$ thermal power, while most of the currentgeneration plants, due to their reliance on the manipulation of the soluble boron concentration, are manually controlled during load-following maneuvers.

However, due to the elimination of the soluble boron adjustment, the perturbations between the global power control subsystem and the axial power distribution control subsystem increase; in other words, the two subsystems are coupled. To solve this problem, an interlock between the two control subsystems is employed, whose principle is that the AO-bank is fixed while the M-banks are moving and the M-banks are fixed while the AObank is moving, and the M-banks have priority. Actually, the moving of the M-backs will also change the $\mathrm{AO}$ value, and the moving of the AO-bank will also change the power level. The coupling between the two subsystems still exists, despite the adoption of the interlock strategy. As a result, the AO control capability is weakened, as is the loadfollowing flexibility of the reactor. Drudy et al. (Drudy et al., 2013) and Wang et al. (Wang et al., 2014) presented and simulated an improved control logic for the AP1000 reactor, which add a logic judgement of the moving directions of the M-banks and AO-bank. If the M-banks and AO-bank need to be moved in the same direction, the M-banks will be stable and the AO-bank will move to adjust both the global power and axial power distributions. The new logic can minimize the potential for interactions between the two control subsystems and guarantee the safety and stability of the reactor core control system. Unfortunately, the coupling is still there. The objective of the present study is to propose a 
decoupling control strategy to eliminate the coupling between the global power and $\mathrm{AO}$ of the AP1000 reactor and cancel the interlocking between the two control subsystems to tighten the AO control and enhance the load-following performance. Digital PID decoupling control based on a quasi-diagonal recurrent neural network (QDRNN) is used in this paper to address this problem.

The remainder of this paper is organized as follows: In section 2, a two-node discrete dynamic model of the AP1000 reactor core is presented. In section 3, the digital PID decoupling controller based on QDRNN is described and applied to the established model. The simulation results are discussed in section 4 . Section 5 concludes the paper.

\section{Dynamic modeling}

In this study, we focus on the global power and axial power distributions of the reactor, so the assumption of a time-independent spatial shape function of the point kinetic model is invalid. For detailed models containing the core physical calculations, thermalhydraulic analysis and burnup optimization are too costly for dynamic simulations of the nuclear reactor because they are very complex and need tremendous calculation work. Therefore, a nodal method with two nodes is used to describe the global power and axial power distributions of the AP1000 reactor core here. The nodes are treated as independent cores coupled with each other through the neutron flux. It is assumed that the neutron flux and material composition in each node are uniform. Thus, the neutron flux and other neutronic parameters in each node are represented by their respective average values integrated over its volume (Wang et al., 2013).

Figure 3 schematically shows the nodalization and neutron diffusion of the two-node reactor model. Node 1 is adopted to illustrate the model, which contains neutron kinetic 
equations, heat transfer equations, xenon kinetic equations, and control rod reactivity equations.

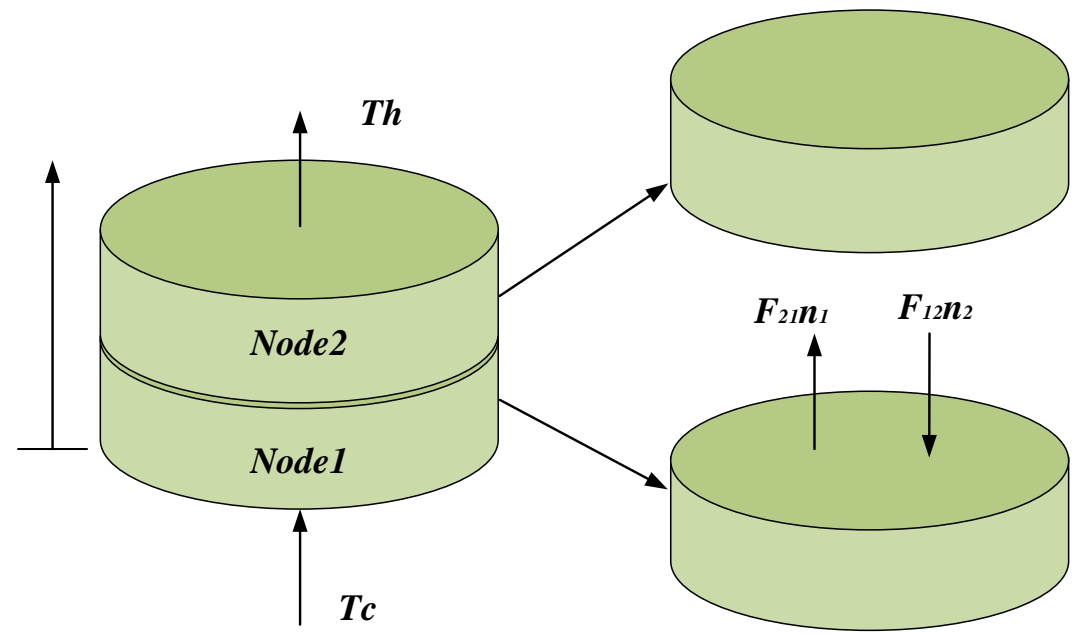

Fig. 3 Nodalization of the two-node AP1000 reactor core in the axial direction. Node 1 is the bottom node, Node 2 is the top node, and $F_{12}$ and $F_{21}$ are the coupling coefficients.

\subsection{Differential equation model}

\section{Neutron Kinetics}

The kinetics of the neutrons includes the prompt neutron generation rate, the delayed neutron generation rate, and the neighbor node neutron diffusion rate. The delayed neutron precursor kinetics includes its generation rate and its decay rate.

The temperature feedback is also very important in the neutron kinetics, so the fuel temperature feedback and the coolant temperature feedback are considered in the calculation of the reactivity. As the active core region of the AP1000 is $4.267 \mathrm{~m}$, which is high enough to consider the xenon kinetics, the xenon concentration feedback is also used in the reactivity calculation,

$$
\begin{gathered}
\frac{d n_{1}}{d t}=\frac{\rho_{1}-\beta}{\Lambda} n_{1}+\sum_{j=1}^{6} \lambda_{j} c_{\mathrm{j} 1}+F_{12} n_{2}-F_{21} n_{1} \\
\frac{d c_{\mathrm{j} 1}}{d t}=\frac{\beta_{j}}{\Lambda} n_{1}-\lambda_{j} c_{\mathrm{j} 1}, j=1,2, \ldots, 6
\end{gathered}
$$

where $n_{1}$ and $n_{2}$ are the neutron concentrations of Node 1 and Node 2 , respectively; $\rho_{1}$ is the reactivity of Node1, calculated as Eq. $3 ; \beta_{j}$ is the fraction of delayed neutrons of the 
$j$ th group, and $\beta$ is the fraction of all the delayed fission neutrons; $\Lambda$ is the average neutron generation time; $\lambda_{j}$ is the decay constant of the $j t h$ delayed neutron; $c_{j 1}$ is the delayed neutron precursor concentration of group $j$ in Node $1 ; F_{12}$ is the coupling coefficient of Node 2 to Node 1 ; and $F_{21}$ is the coupling coefficient of Node 1 to Node 2. The coupling coefficient $F_{12}$ is equal to $F_{21}$ and can be expressed as Eq. 4 .

$$
\rho_{1}=\rho_{r 1}+\alpha_{f 1} \delta T_{f 1}+\alpha_{c 1} \delta T_{c 1}+\alpha_{X 1} \delta n_{X 1}
$$

where $\rho_{r 1}$ is the reactivity provided by the control rods in Node $1 ; \alpha_{f 1}, \alpha_{c 1}$ and $\alpha_{X 1}$ are the fuel reactivity coefficient, coolant reactivity coefficient, and xenon reactivity coefficient of Node 1, respectively; $\delta T_{f 1}, \delta T_{c 1}$, and $\delta n_{X 1}$ are the perturbations of the fuel temperature, coolant temperature, and the xenon concentration around their steady-state values in Node 1, respectively.

$$
F_{12}=F_{21}=-\frac{4 D v}{L^{2}}
$$

where $D$ is the diffusion coefficient; $v$ is the average neutron speed; and $L$ is the height of the core.

\section{Heat transfer}

The fission energy generated in the reactor core appears as heat generated in the nuclear fuel elements, which must be removed to the coolant for the safety of the reactor. Therefore, the heat conduction in the fuel elements and the heat transfer to the coolant are described in the thermal-hydraulics model.

To establish the lumped parameter dynamics of the reactor, the corresponding thermal-hydraulics circuit is also divided into two nodes. As a result, there are four lumps considered, as shown in Fig. 4.

The assumptions of the heat transfer model are as follows: 
(1) The heat transfer model is a single-channel model, in which it is assumed that there is no heat and mass exchange among channels;

(2) Each axial node of the heat transfer model is lumped to have uniform properties such as temperature, pressure and flow rate;

(3) The reactor coolant is at constant density, pressure and mass flow rate, eliminating state equations for mass and momentum.

(4) The axial heat transfer in the fuel is ignored.

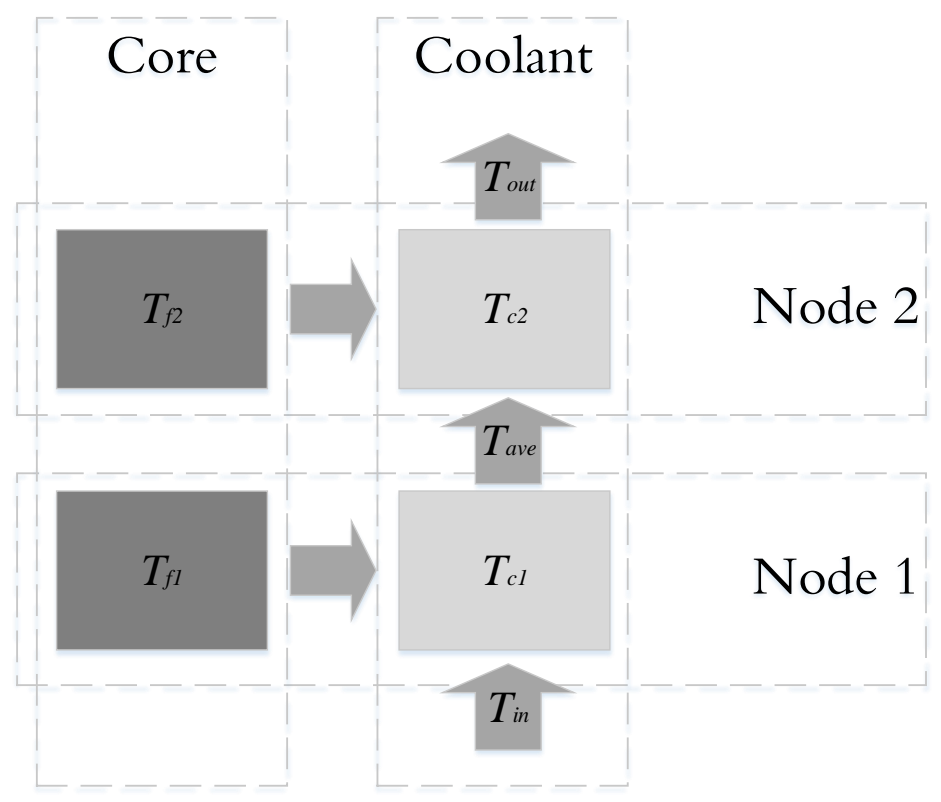

Fig. 4 Schematic of lump relationships for heat transfer model. Node 1 contains the bottom part of the fuel and coolant, whose temperatures are $T_{f 1}$ and $T_{c 1}$, and Node 2 contains the top parts, whose temperatures are $T_{f 2}$ and $T_{c 2}$, respectively. Arrows illustrate the heat transfer direction, $T_{o u t}$ and $T_{\text {in }}$, are the outlet and inlet coolant temperatures, respectively, and $T_{\text {ave }}$ is the average temperature of the coolant assumed at the boundary of Nodes 1 and 2 .

In the fuel part, the energy conservation equation contains the heat generation in the fuel by the fission reaction and the heat transfer from the fuel to the coolant, as Eq.5. 


$$
\begin{aligned}
\frac{d T_{f 1}}{d t} & =\frac{f P_{1}}{\mu_{f 1}}-\frac{\Omega_{1}}{\mu_{f 1}}\left(T_{f 1}-T_{c 1}\right) \\
\mu_{f 1} & =m_{f 1} c_{p f 1}
\end{aligned}
$$

where $T_{f 1}$ and $T_{m 1}$ are the fuel temperature and the average coolant temperature of Node 1 , respectively; $f$ is the fraction of the core power generated in the fuel; $P_{1}$ is the power of Node $1 ; \Omega_{1}$ is the heat transfer coefficient of Node $1 ; m_{f 1}$ is the mass of the fuel; and $c_{p f 1}$ is the specific heat of the fuel.

For the coolant, the energy conservation equation contains the heat generation in the coolant by the fission reaction, the heat transfer from the fuel to the coolant, and the heat convection in this node, as Eq. 7.

$$
\begin{gathered}
\frac{d T_{c 1}}{d t}=\frac{(1-f) P_{1}}{\mu_{c 1}}+\frac{\Omega_{1}}{\mu_{c 1}}\left(T_{f 1}-T_{c 1}\right)-\frac{M_{1}}{\mu_{c 1}}\left(T_{a v e}-T_{i n}\right) \\
\mu_{c 1}=m_{c 1} c_{p c 1} \\
M_{1}=m_{c 1} G_{c} \\
T_{\text {ave }}=\frac{T_{o u t}+T_{\text {in }}}{2}
\end{gathered}
$$

where $m_{c 1}$ is the mass of the coolant; $c_{p c 1}$ is the specific heat of the coolant; $G_{c}$ is the mass flow rate of the coolant; $T_{\text {out }}$ and $T_{\text {in }}$, are the outlet and inlet coolant temperatures, respectively; and $T_{a v e}$ is the temperature at the boundary of Nodes 1 and 2 , which is assumed to be the average of $T_{\text {out }}$ and $T_{\text {in }}$.

\section{Iodine and Xenon kinetics}

Because ${ }^{135} \mathrm{Xe}$ has a large thermal neutron absorption cross-section, it is considered to be the most important isotope in the operation and control of thermal reactors. The scheme of the iodine and xenon kinetics is shown in Fig. 5. A small fraction of this isotope is formed directly through fission, but the major portion originates from the radioactive 
decay of ${ }^{135} \mathrm{I}$, with a half-life of 6-7 hours. Therefore, it is essential to consider the iodine and xenon kinetics in the model.

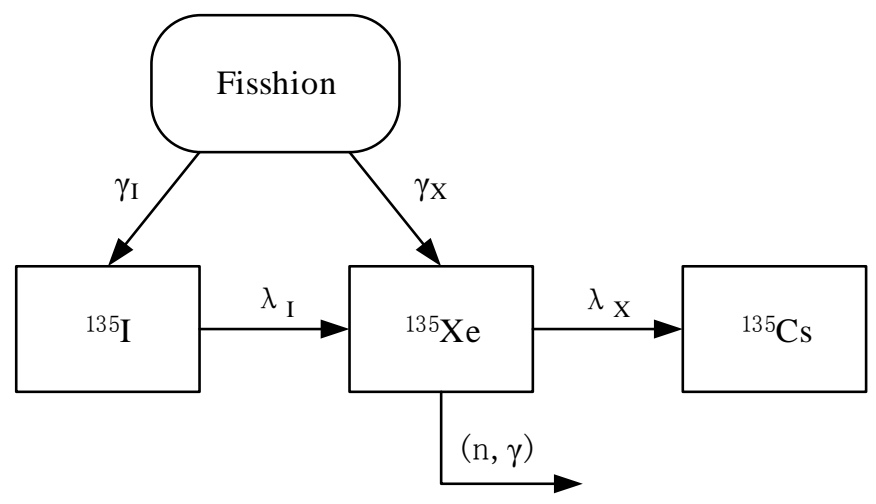

Fig. 5 Schematic diagram of iodine and xenon kinetics in reactor core. $\gamma_{I}$ and $\gamma_{X}$ are the fission yields of iodine and xenon, respectively, $\lambda_{I}$ and $\lambda_{X}$ are the decay constants of iodine and xenon, respectively, and $(n, \gamma)$ denotes the neutron capture reaction.

According to the iodine and xenon kinetics described in Fig. 5, time-based equations for the concentrations of iodine and xenon can be derived,

$$
\begin{gathered}
\frac{d n_{I 1}}{d t}=\gamma_{I} \Sigma_{f} v n_{1}-\lambda_{I} n_{I 1} \\
\frac{d n_{X 1}}{d t}=\gamma_{X} \Sigma_{f} v n_{1}+\lambda_{I} n_{I 1}-\left(\lambda_{X}+\sigma_{X} v n_{1}\right) n_{X 1}
\end{gathered}
$$

where $n_{I 1}$ and $n_{X 1}$ are the iodine and xenon concentrations in Node 1 , respectively; $\Sigma_{f}$ is the macroscopic fission cross-section; $\sigma_{X}$ is the microscopic cross-section for absorption for xenon in Node 1; $\gamma_{I}$ and $\gamma_{X}$ are the fission yields of iodine and xenon, respectively; and $\lambda_{I}$ and $\lambda_{X}$ are the decay constants of iodine and xenon, respectively.

Through the same procedure, the differential equation model of Node 2 can be obtained as Eq. 13-19.

$$
\begin{gathered}
\frac{d n_{2}}{d t}=\frac{\rho_{2}-\beta}{\Lambda} n_{2}+\sum_{j=1}^{6} \lambda_{j} c_{\mathrm{j} 2}+F_{21} n_{1}-F_{12} n_{2} \\
\frac{d c_{\mathrm{j} 2}}{d t}=\frac{\beta_{j}}{\Lambda} n_{2}-\lambda_{j} c_{\mathrm{j} 2}, j=1,2, \ldots, 6
\end{gathered}
$$




$$
\begin{gathered}
\rho_{2}=\rho_{r 2}+\alpha_{f 2} \delta T_{f 2}+\alpha_{c 2} \delta T_{c 2}+\alpha_{X 2} \delta n_{X 2} \\
\frac{d T_{f 2}}{d t}=\frac{f P_{2}}{\mu_{f 2}}-\frac{\Omega_{2}}{\mu_{f 2}}\left(T_{f 2}-T_{c 2}\right) \\
\frac{d T_{c 2}}{d t}=\frac{(1-f) P_{2}}{\mu_{c 2}}+\frac{\Omega_{2}}{\mu_{c 2}}\left(T_{f 2}-T_{c 2}\right)+\frac{M_{2}}{\mu_{c 2}}\left(T_{\text {out }}-T_{\text {ave }}\right) \\
\frac{d n_{I 2}}{d t}=\gamma_{I} \Sigma_{f} v n_{2}-\lambda_{I} n_{I 2} \\
\frac{d n_{X 2}}{d t}=\gamma_{X} \Sigma_{f} v n_{2}+\lambda_{I} n_{I 2}-\left(\lambda_{X}+\sigma_{X} v n_{2}\right) n_{X 2}
\end{gathered}
$$

where the symbols have the same meanings as those for Node 1

\subsection{Control Rods}

The relationship between the integral worth and the rod insertion position of the control banks is nonlinear in the original system. However, for the convenience of calculation here, it is assumed to be a linear relationship. The linear relationship is approximated by inserting the initial rod position in the middle of the core. As a result, the reactivity inserted into the top and bottom of the core by the M-banks is calculated as Eq.20 and 21 ,

$$
\begin{aligned}
& \rho_{M 2}=b_{1} B_{M} \\
& \rho_{M 1}=b_{2} B_{M}
\end{aligned}
$$

where $\rho_{M 1}$ and $\rho_{M 2}$ represent the reactivity inserted by the M-banks into Nodes 1 and 2; $b_{1}$ and $b_{2}$ are constant coefficients; and $B_{M}$ is the insertion position of the $\mathrm{M}$ banks.

As the AO bank is restricted within the top half of the core, it will only affect Node 2's reactivity. The reactivity inserted by the AO bank can be calculated by Eq. 22,

$$
\rho_{A O}=b_{3} B_{A O}
$$

where $\rho_{A O}$ is the reactivity inserted by the AO-bank; $b_{3}$ is a constant coefficient; and $B_{A O}$ is the insertion position of the AO bank. 
Therefore, the reactivity provided to Node 1 and Node 2 by the M-banks and AObank can be calculated by Eq. 23 and 24,

$$
\begin{gathered}
\rho_{r 1}=\rho_{M 1}=b_{2} B_{M} \\
\rho_{r 2}=\rho_{M 2}+\rho_{A O}=b_{1} B_{M}+b_{3} B_{A O}
\end{gathered}
$$

\subsection{Difference Equation model}

Based on the stable-state parameters and the equilibrium considerations, the abovementioned differential equation model can be normalized as Eq. 25-37,

$$
\text { Let } N_{1}=\frac{n_{1}}{n_{1,0}}, C_{j 1}=\frac{c_{j 1}}{c_{j 1,0}}, I o_{1}=\frac{n_{I 1}}{n_{I 1,0}}, X e_{1}=\frac{n_{X 1}}{n_{X 1,0}}, N_{2}=\frac{n_{2}}{n_{2,0}}, C_{j 2}=\frac{c_{j 2}}{c_{j 2,0}}, I o_{2}=\frac{n_{I 2}}{n_{I 2,0}} \text {, }
$$

and $X e_{2}=\frac{n_{X 2}}{n_{X 2,0}}$.

$$
\begin{gathered}
\frac{d N_{1}}{d t}=\left[\frac{1}{\Lambda}\left(b_{2} B_{M}+\alpha_{f 1} \delta T_{f 1}+\alpha_{c 1} \delta T_{c 1}+\alpha_{X 1} n_{X 1,0} \delta X e_{1}-\beta\right)-F_{12}\right] N_{1}+F_{21} \frac{n_{2,0}}{n_{1,0}} N_{2}+ \\
\sum_{j=1}^{6} \frac{\beta_{j}}{\Lambda} C_{\mathrm{j} 1} \\
\frac{d C_{j 1}}{d t}=\lambda_{j} N_{1}-\lambda_{j} C_{j 1}, \quad j=1,2, \ldots, 6 \\
\frac{d T_{f 1}}{d t}=\frac{f P_{1,0}}{\mu_{f 1}} N_{1}-\frac{\Omega_{1}}{\mu_{f 1}} T_{f 1}+\frac{\Omega_{1}}{\mu_{f 1}} T_{c 1} \\
\frac{d T_{c 1}}{d t}=\frac{(1-f) P_{1,0}}{\mu_{c 1}} N_{1}+\frac{\Omega_{1}}{\mu_{c 1}} T_{f 1}-\frac{2 \mathrm{M}_{1}+\Omega_{1}}{\mu_{c 1}} T_{c 1}+\frac{2 \mathrm{M}_{1}}{\mu_{c 1}} T_{i n} \\
\frac{d I o_{1}}{d t}=\gamma_{I} \Sigma_{f} v \frac{n_{1,0}}{n_{\mathrm{I} 1,0}} N_{1}-\lambda_{I} I o_{1} \\
\frac{d X e_{1}}{d t}=\gamma_{X} \sum_{f} v \frac{n_{1,0}}{n_{\mathrm{I} 1,0}} N_{1}-\lambda_{X} X e_{1}+\lambda_{I} \frac{n_{\mathrm{I} 1,0}}{n_{1,0}} I o_{1}-\sigma_{X} v n_{1,0} X e_{1} N_{1} \\
\frac{d N_{2}}{d t}=\left[\frac{1}{\Lambda}\left(b_{1} B_{M}+b_{3} B_{A O}+\alpha_{f 2} \delta T_{f 2}+\alpha_{c 2} \delta T_{c 2}+\alpha_{X 2} n_{X 2,0} \delta X e_{2}-\beta\right)-F_{21}\right] N_{2}+ \\
F_{12} \frac{n_{1,0}}{n_{2,0}} N_{1}+\sum_{j=1}^{6} \frac{\beta_{j}}{\Lambda} C_{\mathrm{j} 2} \\
C_{\mathrm{j} 2}=\lambda_{j} N_{2}-\lambda_{j} C_{\mathrm{j} 2}
\end{gathered}
$$




$$
\begin{gathered}
\frac{d T_{f 2}}{d t}=\frac{f P_{2,0}}{\mu_{f 2}} N_{2}-\frac{\Omega_{2}}{\mu_{f 2}} T_{f 2}+\frac{\Omega_{2}}{\mu_{f 2}} T_{c 2} \\
\frac{d T_{c 2}}{d t}=\frac{(1-f) P_{2,0}}{\mu_{c 2}} N_{2}+\frac{\Omega_{2}}{\mu_{c 2}} T_{f 2}-\frac{2 \mathrm{M}_{2}+\Omega_{2}}{\mu_{c 2}} T_{c 2}+\frac{2 \mathrm{M}_{2}}{\mu_{c 2}} T_{a v e} \\
\frac{d I o_{2}}{d t}=\gamma_{I} \Sigma_{f} v \frac{n_{2,0}}{n_{\mathrm{I} 2,0}} N_{2}-\lambda_{I} I o_{2} \\
\frac{d X e_{2}}{d t}=\gamma_{X} \Sigma_{f} v \frac{n_{2,0}}{n_{\mathrm{I} 2,0}} N_{2}-\lambda_{X} X e_{2}+\lambda_{I} \frac{n_{\mathrm{I} 2,0}}{n_{2,0}} I o_{2}-\sigma_{X} v n_{1,0} X e_{2} N_{2}
\end{gathered}
$$

where $N, C_{j}, I o$, and $X e$ are the normalized values of $n, c_{j}, n_{I}$, and $n_{X}$; subscripts 1 and 2 indicate Nodes 1 and 2; and subscript 0 indicates a steady-state value.

To use the digital proportion integration differentiation (PID) control, the model should be dispersed. Using $\tau$ as the sampling period, the difference equation model of the reactor core can be expressed as Eq. 37-48.

$$
\begin{gathered}
N_{1}(k+1)=\left\{\left\{\frac { \tau } { \Lambda } \left\{b_{2} B_{M}(k)+\alpha_{f 1}\left[T_{f 1}(k)-T_{f 1}(k-1)\right]+\alpha_{c 1}\left[T_{c 1}(k)-T_{c 1}(k-1)\right]+\right.\right.\right. \\
\left.\left.\left.\alpha_{X 1} n_{X 1,0}\left[X e_{1}(k)-X e_{1}(k-1)\right]-\beta\right\}-F_{12}\right\}+1\right\} N_{1}(k)+F_{21} \frac{n_{2,0}}{n_{1,0}} \tau N_{2}(k)+ \\
C_{\mathrm{j} 1}(k+1)=\lambda_{j} \tau N_{1}(k)-\left(\lambda_{j} \tau+1\right) C_{j=1} \frac{\beta_{j}}{\Lambda} C_{\mathrm{j} 1}(k), \quad j=1,2, \ldots, 6 \\
T_{f 1}(k+1)=\frac{f P_{1,0}}{\mu_{f 1}} \tau N_{1}(k)-\left(\frac{\Omega_{1}}{\mu_{f 1}} \tau-1\right) T_{f 1}(k)+\frac{\Omega_{1}}{\mu_{f 1}} \tau T_{c 1}(k) \\
T_{c 1}(k+1)=\frac{(1-f) P_{1,0}}{\mu_{c 1}} \tau N_{1}(k)+\frac{\Omega_{1}}{\mu_{c 1}} \tau T_{f 1}(k)-\left(\frac{2 \mathrm{M}_{1}+\Omega_{1}}{\mu_{c 1}} \tau-1\right) T_{c 1}(k)+\frac{2 \mathrm{M}_{1}}{\mu_{c 1}} \tau T_{i n}(k) \\
I o_{1}(k+1)=\gamma_{I} \Sigma_{f} v \frac{n_{1,0}}{n_{\mathrm{I} 1,0}} \tau N_{1}(k)-\left(\lambda_{I} \tau-1\right) I o_{1}(k) \\
X e_{1}(k+1)=\gamma_{X} \Sigma_{f} v \frac{n_{1,0}}{n_{\mathrm{I} 1,0}} \tau N_{1}(k)-\left(\lambda_{X} \tau-1\right) X e_{1}(k)+\lambda_{I} \frac{n_{\mathrm{I} 1,0}}{n_{1,0}} \tau I o_{1}(k)-
\end{gathered}
$$




$$
\begin{gathered}
N_{2}(k+1)=\left\{\left\{\frac { \tau } { \Lambda } \left\{b_{1} B_{M}(k)+b_{3} B_{A O}(k)+\alpha_{f 2}\left[T_{f 2}(k)-T_{f 2}(k-1)\right]+\alpha_{c 2}\left[T_{c 2}(k)-\right.\right.\right.\right. \\
\left.\left.\left.\left.T_{c 2}(k-1)\right]+\alpha_{X 2} n_{X 2,0}\left[X e_{2}(k)-X e_{2}(k-1)\right]-\beta\right\}-F_{21}\right\}+1\right\} N_{2}(k)+ \\
F_{12} \frac{n_{1,0}}{n_{2,0}} \tau N_{1}(k)+\tau \sum_{j=1}^{6} \frac{\beta_{j}}{\Lambda} C_{\mathrm{j} 2}(k) \\
C_{\mathrm{j} 2}(k+1)=\lambda_{j} \tau N_{2}(k)-\left(\lambda_{j} \tau+1\right) C_{\mathrm{j} 2}(k), \quad j=1,2, \ldots, 6 \\
T_{f 2}(k+1)=\frac{f P_{2,0}}{\mu_{f 2}} \tau N_{2}(k)-\left(\frac{\Omega_{2}}{\mu_{f 2}} \tau-1\right) T_{f 2}(k)+\frac{\Omega_{2}}{\mu_{f 2}} \tau T_{c 2}(k) \\
\begin{array}{c}
\frac{(1-f) P_{2,0}}{\mu_{c 2}} \tau N_{2}(k)+\frac{\Omega_{2}}{\mu_{c 2}} \tau T_{f 2}(k)-\left(\frac{2 \mathrm{M}_{2}+\Omega_{2}}{\mu_{c 2}} \tau-1\right) T_{c 2}(k)+\frac{2 \mathrm{M}_{2}}{\mu_{c 2}} \tau T_{i n}(k) \\
I o_{2}(k+1)=\gamma_{I} \Sigma_{f} v \frac{n_{2,0}}{n_{\mathrm{I} 2,0}} \tau N_{2}(k)-\left(\lambda_{I} \tau-1\right) I o_{2}(k) \\
X e_{2}(k+1)=\gamma_{X} \sum_{f} v \frac{n_{2,0}}{n_{\mathrm{I} 2,0}} \tau N_{2}(k)-\left(\lambda_{X} \tau-1\right) X e_{2}(k)+\lambda_{I} \frac{n_{\mathrm{I} 2,0}}{n_{2,0}} \tau I o_{2}(k)- \\
\sigma_{X} v n_{2,0} \tau X e_{2} N_{2}(k)
\end{array}
\end{gathered}
$$

The global power and the axial power distributions are the focuses of this study and can be calculated by Eq. 49 and 50,

$$
\begin{gathered}
P(k)=\frac{N_{1}(k)+N_{2}(k)}{2} \\
A O(k)=\frac{N_{1}(k)-N_{2}(k)}{N_{1}(k)+N_{2}(k)} \times 100 \%
\end{gathered}
$$

where $P$ is the global power, and $A O$ is the axial power offset.

\section{Decoupling control of the Ap1000 reactor core}

With its simpler algorithm, better robustness, and higher reliability, the digital PID controller is widely used in industrial control systems (Liu, 2011; Han, 2012). Therefore, the digital PID controller is used to decouple the coupled AP1000 reactor core system in this study. As is known, the parameter tuning of the PID controller is the most important 
aspect of the system design. Considering its advantages of online tuning and high robustness, a type of neural network named the diagonal recurrent neural network (QDRNN) is used to tune the parameters of the digital PID controller here ( $\mathrm{Li}, 2004,2007)$.

\subsection{Diagonal recurrent neural network (QDRNN)}

The QDRNN retains the simple structure of the diagonal recurrent neural network (DRNN) to decrease the computational requirements, but the connections between adjacent recurrent neutrons are added (Li, 2004; Dray, 1996). The QDRNN's structure is shown in Fig. 6, consisting of one input layer, one hidden layer, and one output layer. The hidden layer, which is the recurrent layer, builds the relationships between neighboring neurons.

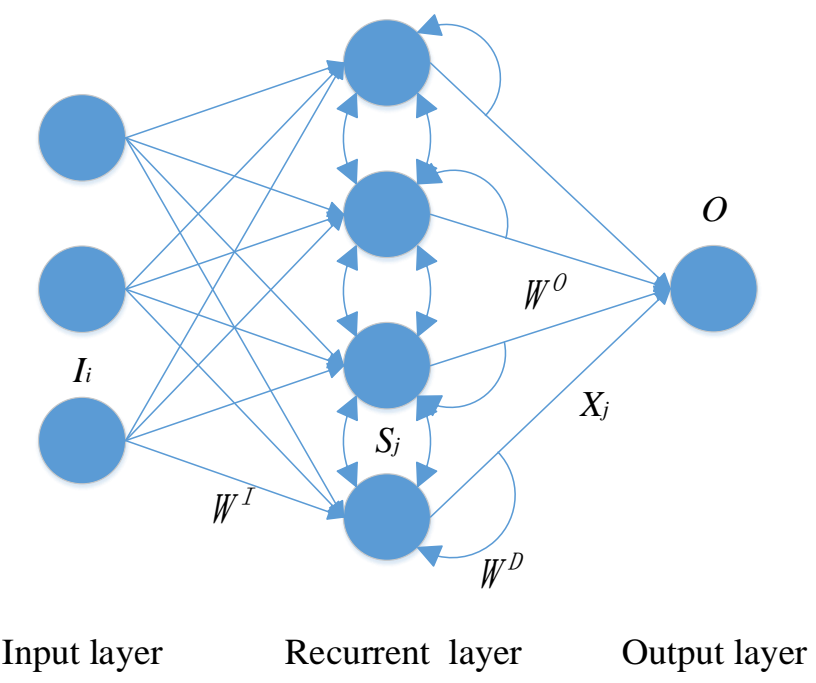

Fig. 6 Structure of QDRNN.

$I_{i}$ is the input of the $i t h$ neuron in the input layer, $S_{j}$ is the input summation of the $j t h$ neuron in the recurrent layer, $X_{j}$ is the output of the $j$ th neuron in the recurrent layer, $O$ is the output of the network, and $W^{I}, W^{O}$, and $W^{D}$ are the connection weight matrices between the input layer and the recurrent layer, between the recurrent layer and the output layer, and within the recurrent layer, respectively. 
It can be seen in Fig. $6, I=\left[I_{1}, I_{2}, \ldots, I_{n}\right]$ is the input of the QDRNN, $I_{i}$ is the input of the ith neuron of the input layer. $X_{j}$ is the output of the $j$ th neuron of the recurrent layer. $S_{j}$ is the input summation of the $j$ th neuron in recurrent layer. $O$ is the output of the network.

Figure 7 is the identification process of QDRNN. $k$ is the iterative step of QDRNN, $u(k)$ is the input of the object. $y(k)$ is the real output of the object. Take $u(k)$ and $y(k)$ as the the inputs of the QDRNN, $y m(k)$ is the identification output of QDRNN. The error of $y(k)$ and $y m(k)$ is used for identifying error.

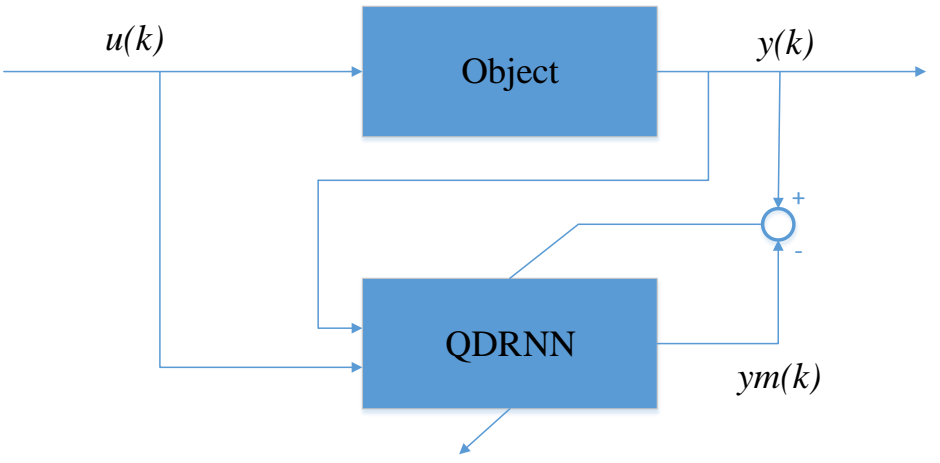

Fig. 7 The identifying process of QDRNN.u(k) is the input of the object, $y(k)$ is the real output of the object, $y m(k)$ is the identification output of QDRNN.

The algorithm of QDRNN can be illustrated as follow.

The output of the output layer,

$$
y m(k)=O(k)=\sum_{j} W_{j}^{O} X_{j}(k)
$$

The output of the recurrent layer,

$$
X_{j}(k)=f\left[S_{j}(k)\right]
$$

The input of the recurrent layer,

$$
S_{j}(k)=W_{j}^{D} X_{j}(k-1)+\sum_{i} W_{i j}^{I} I_{i}(k)
$$


where the recurrent connection weight matrix $\left(W^{D}\right)$ is a quasi-diagonal matrix as Eq.54, and the activation function is a sigmoid function as Eq.55.

$$
\begin{gathered}
W^{D}=\left[\begin{array}{ccccccc}
w_{11}^{d} & w_{12}^{d} & & & \cdots & 0 & \\
w_{21}^{d} & w_{22}^{d} & w_{23}^{d} & & & & \\
\vdots & \vdots & \vdots & \vdots & \vdots & \vdots & \vdots \\
& & & & w_{(n-1)(n-2)}^{d} & w_{(n-1)(n-1)}^{d} & w_{(n-1) n}^{d} \\
& 0 & & \ldots & & w_{n(n-1)}^{d} & w_{n n}^{d}
\end{array}\right] \\
\\
\end{gathered}
$$

The identifying error of QDRNN,

$$
\Delta y(k)=y(k)-y m(k)
$$

The identifying index of QDRNN,

$$
J=\frac{1}{2}\|\Delta y(k)\|^{2}
$$

The connection weight matrices can be modified by the gradient-descent algorithm as Eq.58-65.

Output layer weight modification,

$$
\begin{gathered}
\Delta w_{j}^{O}(k)=-\frac{\partial J}{\partial w_{j}^{O}}=\Delta O(k) X_{j}(k) \\
w_{j}^{O}(k)=w_{j}^{O}(k-1)+\mu_{O} \Delta w_{j}^{O}(k)+\alpha\left[w_{j}^{O}(k-1)-w_{j}^{O}(k-2)\right]
\end{gathered}
$$

Input layer weight modification,

$$
\begin{gathered}
\Delta w_{i j}^{I}(k)=-\frac{\partial J}{\partial w_{i j}^{I}}=\Delta O(k) w_{j}^{O} Q_{i j}(k) \\
w_{i j}^{I}(k)=w_{i j}^{I}(k-1)+\mu_{I} \Delta w_{i j}^{I}(k)+\alpha\left[w_{i j}^{I}(k-1)-w_{i j}^{I}(k-2)\right] \\
Q_{i j}(k)=\frac{\partial X_{j}}{\partial w_{i j}^{I}}=f^{\prime}\left(S_{j}\right) I_{i}(k)
\end{gathered}
$$

Recurrent layer weight modification

$$
\Delta w_{j}^{D}(k)=-\frac{\partial J}{\partial w_{j}^{D}}=\Delta O(k) w_{j}^{O} P_{j}(k)
$$




$$
\begin{gathered}
w_{j}^{D}(k)=w_{j}^{D}(k-1)+\mu_{D} \Delta w_{j}^{D}(k)+\alpha\left[w_{j}^{D}(k-1)-w_{j}^{D}(k-2)\right] \\
P_{j}(k)=\frac{\partial X_{j}}{\partial w_{j}^{D}}=f^{\prime}\left(S_{j}\right) X_{j}(k-1)
\end{gathered}
$$

where $\mu_{I}, \mu_{O}$, and $\mu_{D}$ are the learning rates of the input layer, output layer, and recurrent

layer $\alpha$ is the inertia coefficient. Jacobian information of the object is $\frac{\partial y}{\partial u}$, which can be calculated as Eq. 66.

$$
\frac{\partial y}{\partial \mathrm{u}} \approx \frac{\partial y m}{\partial \mathrm{u}}=\sum_{j} w_{j}^{O} f^{\prime}\left(S_{j}\right) w_{i j}^{I}
$$

\subsection{Decoupling design by digital PID controller based on QDRNN}

As the AP1000 reactor core has two inputs of the power control subsystem and the axial offset control subsystem and two outputs of the global power and $\mathrm{AO}$, the reference values of the global power and $\mathrm{AO}$ are taken as the disturbances of the system. Two digital PID controllers are used in the decoupling control system, and two QDRNNs are used to tune the PID parameters online. Figure 8 is the decoupling control system designed by the digital PID controller based on QDRNN. 


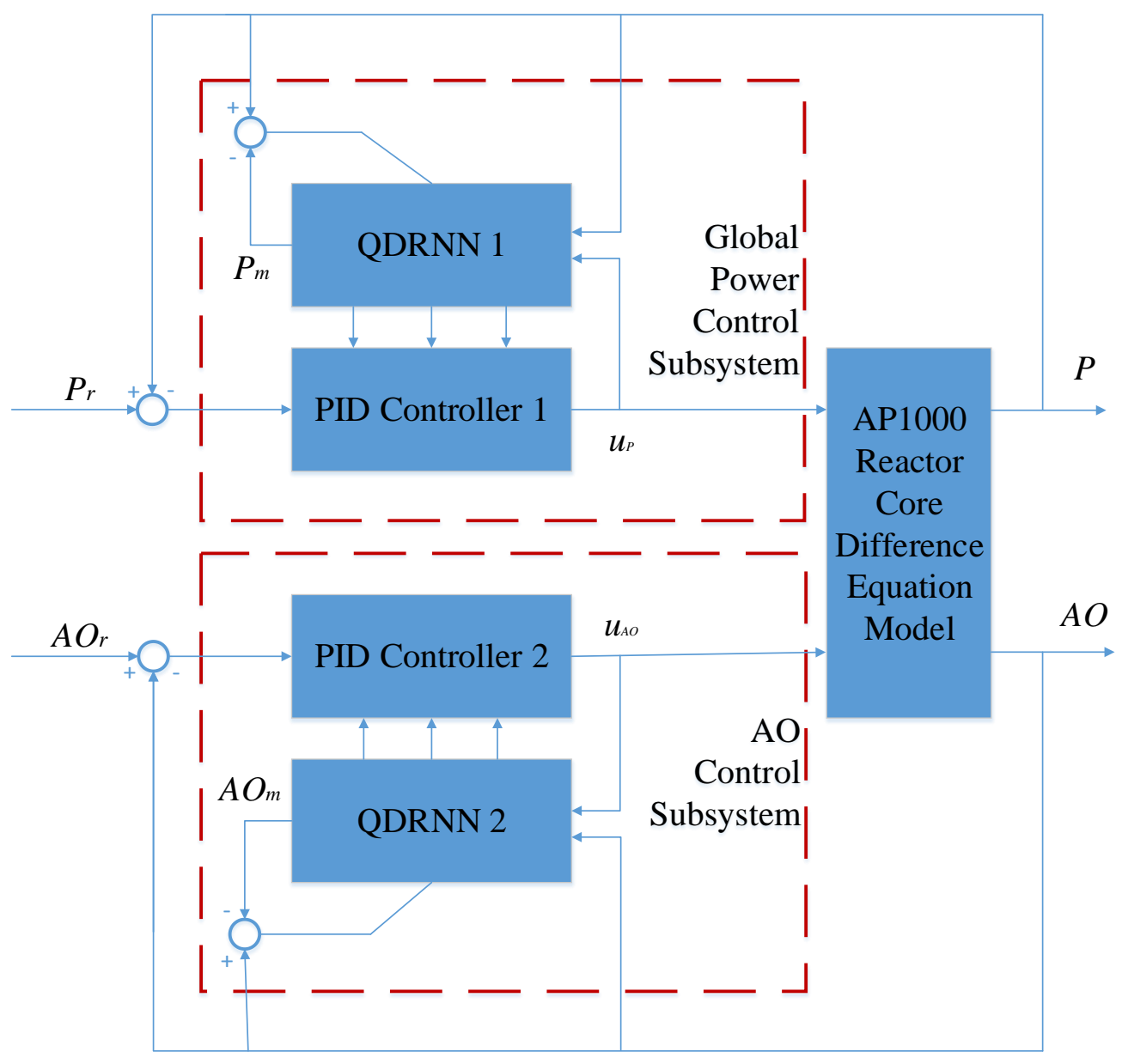

Fig. 8 Structure of the decoupling control system of the AP1000 reactor core by digital PID control based on QDRNN. $P$ and $A O$ are the two outputs of the system and the inputs $P_{r}$ and $A O_{r}$ are the reference values of $P$ and $A O$, respectively, $u_{P}$ and $u_{A O}$ are the two control outputs of the two PID controllers, $P_{m}$ and $A O_{m}$ are the identifying valves of $P$ and $A O$ by QDRNN.

In Fig.8, the PID Controller 1 and PID Controller 2 are the digital PID controller for global power control subsystem and $\mathrm{AO}$ control subsystem respectively $P$ and $A O$ are the two outputs of the system, the inputs $P_{r}$ and $A O_{r}$ are the reference valves of $P$ and $A O$, respectively. $u_{P}$ and $u_{A O}$ are the two control outputs of the two PID controllers. $P_{m}$ and $A O_{m}$ are the identifying valves of $P$ and $A O$ by QDRNN, respectively. The parameters of the two controllers are $k_{p, P}, k_{i, P}, k_{d, P}, k_{p, A O}, k_{i, A O}, k_{d, A O}$ which are tuned online by QDRNN 1 and QDRNN 2 as Eq.72-74. 


$$
\begin{aligned}
& u(k)=k_{p}(k) x_{1}(k)+k_{i} x_{2}(k)+k_{d} x_{3}(k) \\
& x_{1}(k)=\operatorname{error}(k) \\
& x_{2}(k)=\sum_{i=1}^{k} \operatorname{error}(k) \times \tau \\
& x_{3}(k)=\frac{\operatorname{error}(k)-\operatorname{error}(k-1)}{\tau} \\
& E(k)=\frac{1}{2}[\operatorname{error}(k)]^{2} \\
& k_{p}(k)=k_{p}(k-1)-\eta_{p} \frac{\partial E}{\partial k_{p}}=k_{p}(k-1)+\eta_{p} \operatorname{error}(k) \frac{\partial y}{\partial u} x_{1}(k) \\
& k_{i}(k)=k_{i}(k-1)-\eta_{i} \frac{\partial E}{\partial k_{i}}=k_{i}(k-1)+\eta_{i} \operatorname{error}(k) \frac{\partial y}{\partial u} x_{2}(k) \\
& k_{d}(k)=k_{d}(k-1)-\eta_{d} \frac{\partial E}{\partial k_{d}}=k_{d}(k-1)+\eta_{d} \operatorname{error}(k) \frac{\partial y}{\partial u} x_{3}(k) \\
& \operatorname{error}_{P}(k)=P_{r}(k)-P(k) \\
& \operatorname{error}_{A O}(k)=A O_{r}(k)-A O(k) \\
& E_{P}(k)=\frac{1}{2}\left[\operatorname{error}_{P}(k)\right]^{2} \\
& E_{A O}(k)=\frac{1}{2}\left[\operatorname{error}_{A O}(k)\right]^{2}
\end{aligned}
$$

where $k_{p}, k_{i}$, and $k_{d}$ are the parameters of the PID controllers; error is the error of the calculation value and reference value; $E$ is the parameter turning performance objectives of the PID controllers; subscripts $P$ and $A O$ indicate the global power control subsystem and AO control subsystem respectively. $\frac{\partial y}{\partial u}$ is the Jacobian information of the system, which can be identified by QDRNN as Eq.66. In other words, the parameters of the PID controllers can be tuned online in this way.

\section{Simulation results and discussion}

Based on the difference equation model established in Section 2 and the decoupling control system designed in Section3, the system is simulated in this section. The connection 
weight matrices of the input layer, output layer, and recurrent layer in the neural networks are all random matrices as Eq.79. In consideration of the convergence rate and the stability of the system, the learning rates and the inertia coefficients are set as Eq.80 and Eq. 81 respectively.

$$
\begin{aligned}
& \left\{\begin{array}{c}
W_{P}^{I}=\operatorname{rands}(3,5), W_{P}^{D}=\operatorname{rands}(5,5), W_{P}^{O}=\operatorname{rand}(5,1) \\
W_{A O}^{I}=\operatorname{rands}(3,5), W_{A O}^{D}=\operatorname{rands}(5,5), W_{A O}^{O}=\operatorname{rands}(5,1)
\end{array}\right. \\
& \left\{\begin{array}{c}
\eta_{p, P}=0.5, \eta_{i, P}=0.5, \eta_{d, P}=0.5 \\
\eta_{p, A O}=0.5, \eta_{i, A O}=0.5, \eta_{d, A O}=0.5
\end{array}\right. \\
& \left\{\begin{array}{c}
\alpha_{P}=0.04 \\
\alpha_{A O}=0.04
\end{array}\right.
\end{aligned}
$$

\subsection{Decoupling performance analysis}

To analyze the effect of the designed decoupling control system, the performances of the original control system and decoupling control system are simulated in this section. As is known, the step signal indicates the strictest condition of the processing. This means that if the decoupling system works and is reliable under a step signal, it will work reliably under other conditions. A 10\% step decrease signal is provided to the two subsystems for testing the responses of $P$ and $A O$. The test condition is illustrated as Condition 1, and the simulation results are showed in Fig. 9 -10.

Condition 1 (10\% step reduce to $P_{r}$ and constant $\left.A O_{r}\right)$ : Starting from $200^{\text {th }}$ seconds, the global power reference value $\left(P_{r}\right)$ steps down from $100 \%$ to $90 \%$ reactor full power (RFP) and AO reference valve $\left(A O_{r}\right)$ remain unchanged at 0. 


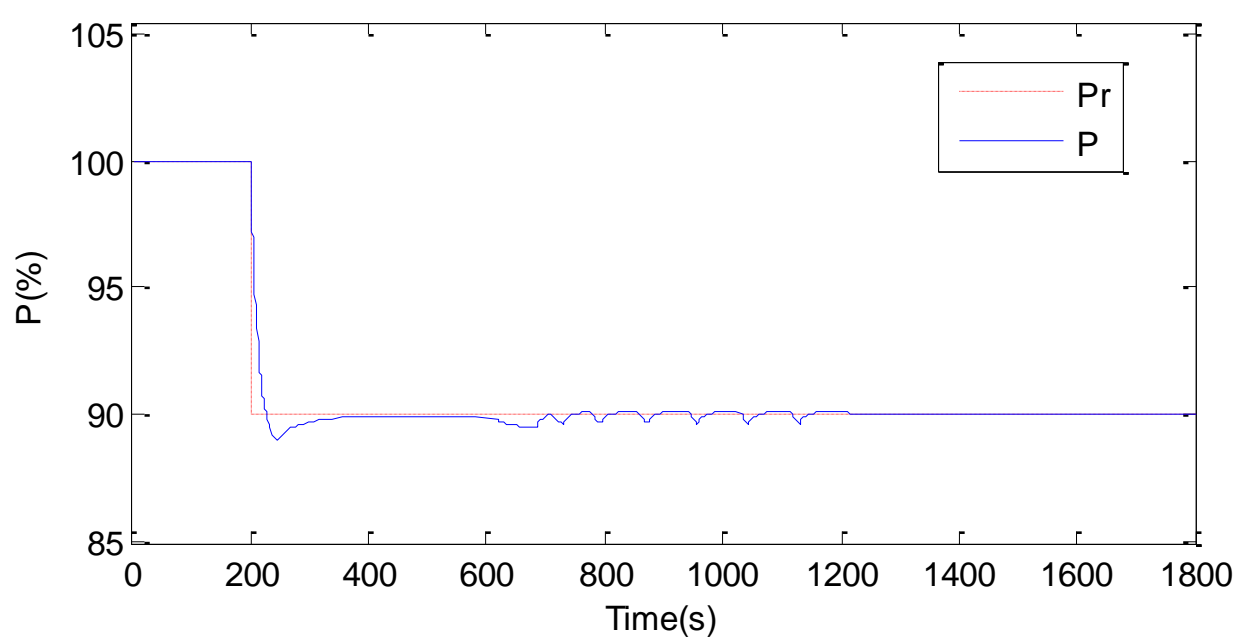

(a)

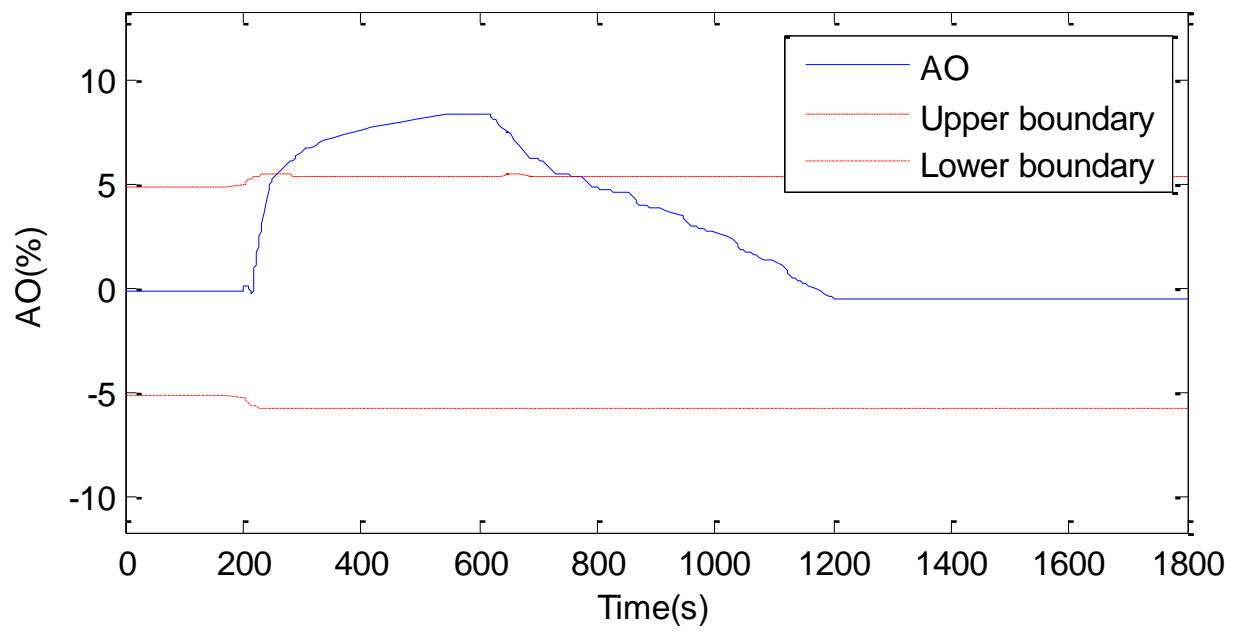

(b)

Fig. 9 Original control system simulation results of Condition 1. (a)P, (b) AO. 


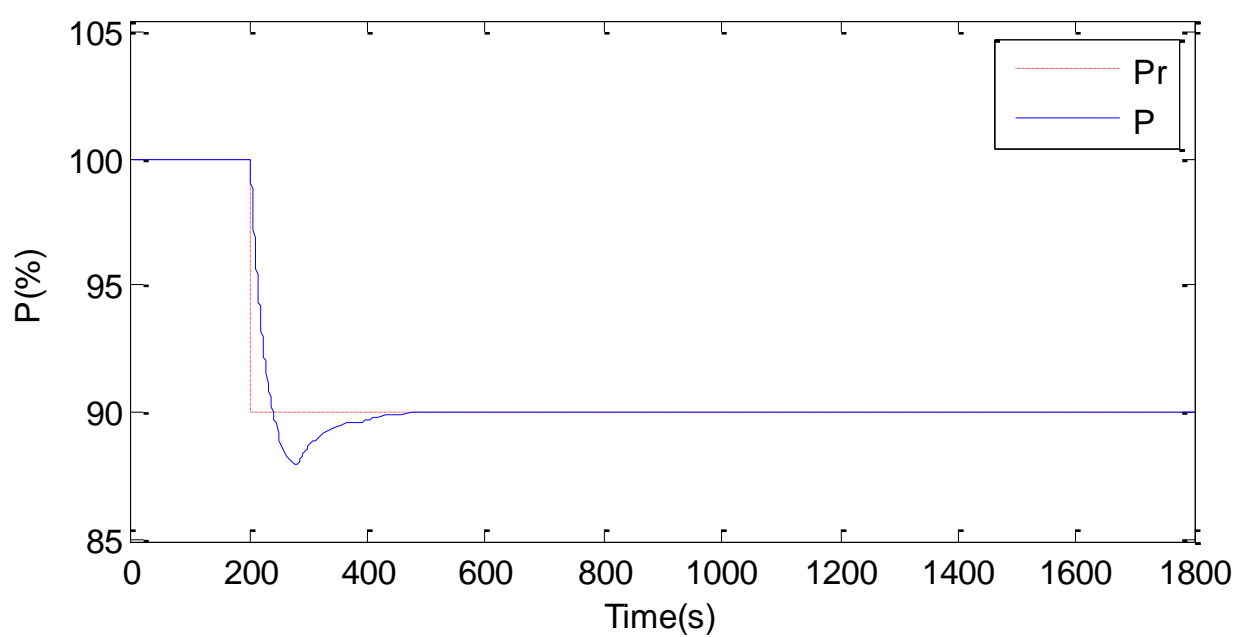

(a)

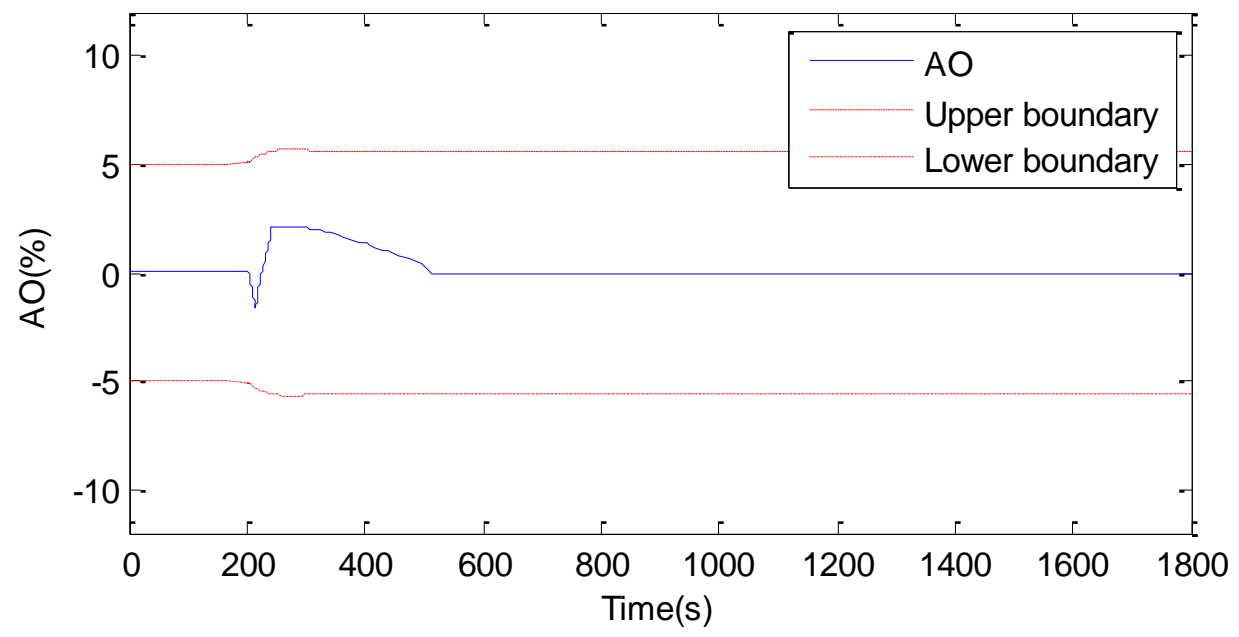

(b)

Fig. 10 Decoupling control system simulation results of Condition 1. (a)P, (b) AO.

From Fig. 9 and Fig. 10, it can be seen that the settling time of the original system is approximately 1000 seconds, which is much longer than the 300 seconds of the decoupling control system. Additionally, P in Fig. 9(a) appears as a zigzag shape, and AO in Fig. 9(b) appears as a stair shape. The reason is the interlock strategy between the M-banks and AO-bank; in other words, when $\mathrm{P}$ is adjusted in its deadband by the M-banks, AO is able to be adjusted by the AO-bank. However, in the process of the AO 
adjustment, $\mathrm{P}$ will also be changed, and when $\mathrm{P}$ gets out of its deadband again, the AObank will be locked by the movement signal of the M-banks. In the original system, the AO's maximum overshoot is much higher because the AO-bank can only move in the case of static M-banks, and the movement of the AO-bank will also produce extra reactivity that can change the global power. As a result, the settling time of $\mathrm{P}$ and $\mathrm{AO}$ in the original system is longer than in the decoupling system. In Fig. 10(a), the maximum overshoot of $\mathrm{P}$ is larger than that in Fig. 9(a). The reason is that at the beginning of the scenario, the M-banks and AO-bank can move together and input more reactivity into the decoupling control system. In this situation, the designed decoupling control system can produce tighter and faster control of both $\mathrm{P}$ and $\mathrm{AO}$,

The simulation results show that the designed system can successfully decouple the AP1000 reactor core system. The M-banks and AO-bank are regulated when needed instead of being controlled by an interlock strategy using a digital PID controller whose parameters are tuned by QDRNN. As the tuning of the PID's parameters is online, the robustness is also guaranteed in the designed control system.

\subsection{Load-following operation}

The decoupling control designed is successful according the results of section 4.1. A load-following condition is simulated in this section to assess the feasibility and performance of the designed controller under operation conditions similar to those typical of an actual plant. 
Condition 2 (100-50-100\%, 12-3-6-3 h pattern of daily load-follow): After $100 \%$ RFP steady state operation, $P_{r}$ varies from $100 \%$ to $50 \%$ RFP in $3 \mathrm{~h}$, holds at $50 \% \mathrm{RFP}$ for $6 \mathrm{~h}$, and then rises to $100 \%$ for $3 \mathrm{~h}$ and repeats. $A O$ has an initial value of 0 , a reference value of $-1 \%$, and should be maintained within its target band of $\pm 5 \%$.

Figure 11,and 12 are the simulation results of Condition 2.

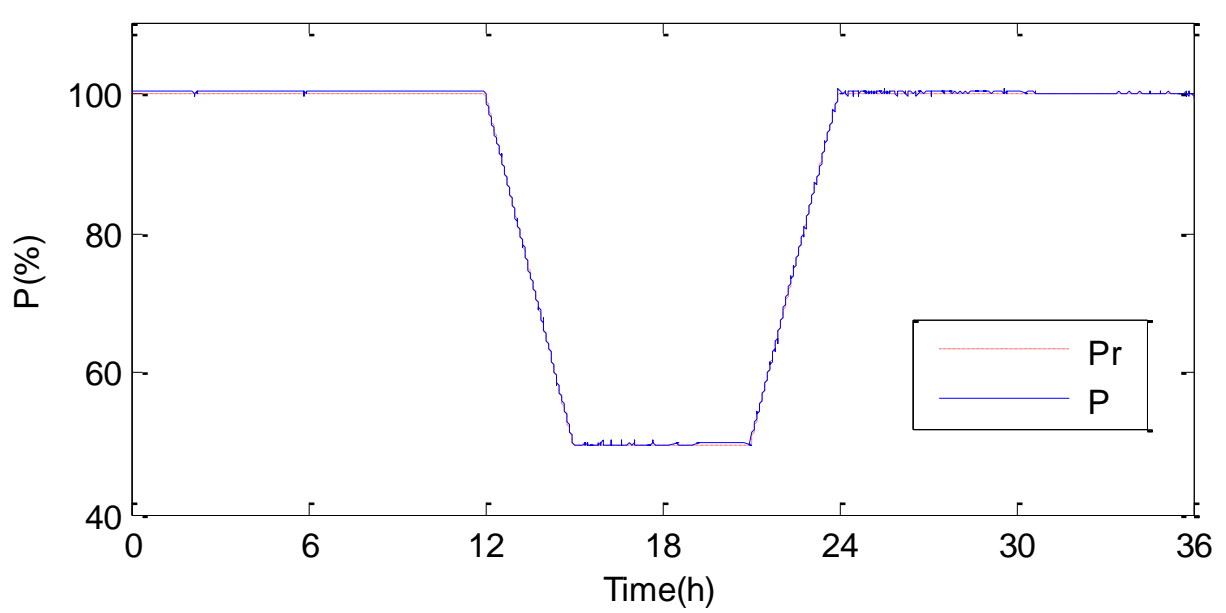

(a)

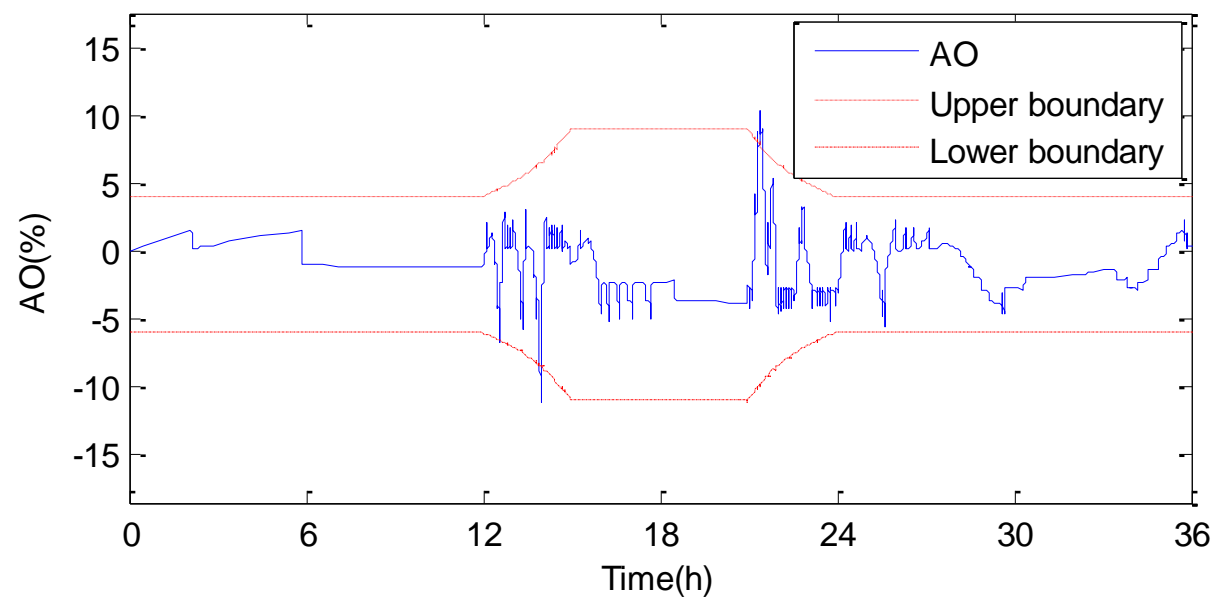

(b)

Fig. 11 Original control system simulation results of Condition 2. (a)P, (b) AO. 


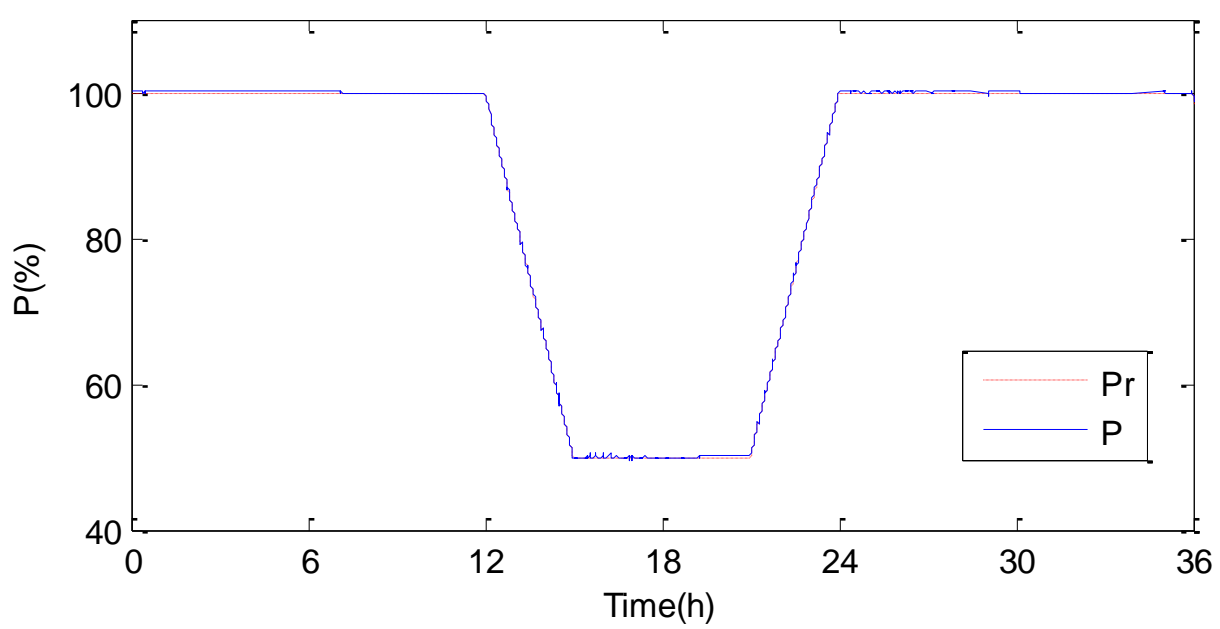

(a)

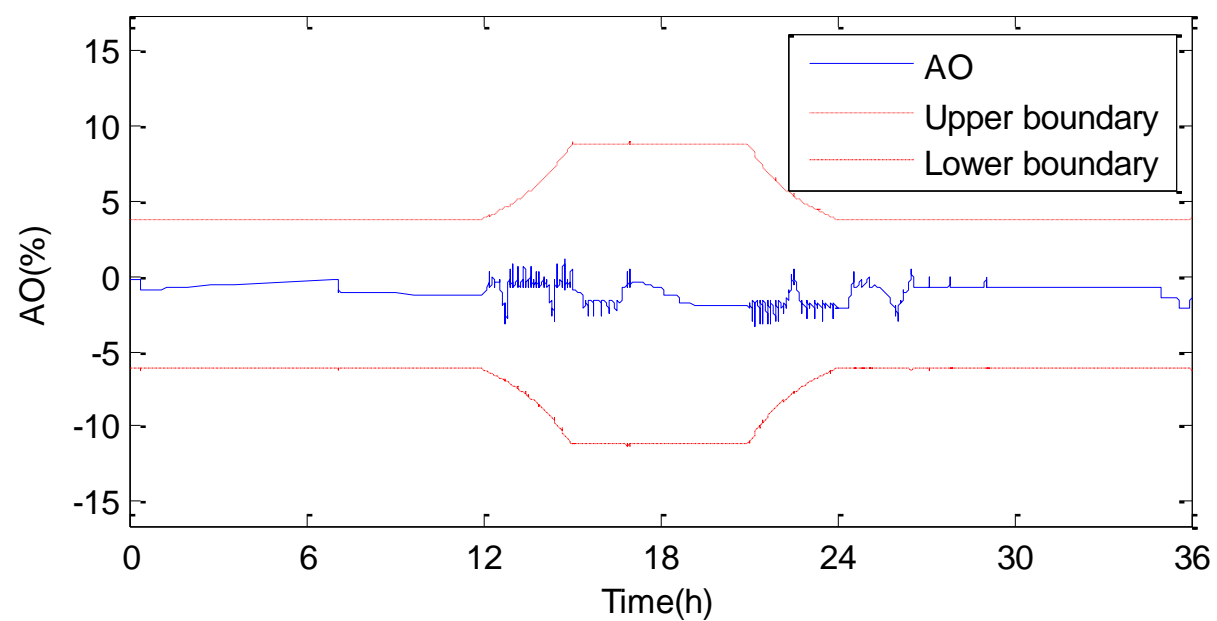

(b)

Fig. 12 Decoupling control system simulation results of Condition 2. (a)P, (b) AO.

The load-following operation is actually a combination of ramp signals. The practicability and reliability under ramp signals indicates the ability of the designed decoupling control system to handle continuous signals. From Fig. 11 and Fig. 12, it can be seen that $P$ follows its $P_{r}$ well for both the original and the decoupling system. However, the AO exceeds its target band around its reference value during the load decrease or increase transients for the original system, as shown in Fig. 11(b). This is 
because the AO-bank is locked when the power control subsystem moves the Mbanks to regulate $P$. Figure 12(b) shows that the AO is tightly controlled within the target band during the power maneuvering in the decoupling system. This is because the M-banks and AO-bank are allowed to move at the same time under the control of the global power control subsystem and AO control subsystem. Thus, the designed decoupling control system can provide much tighter AO control without compromising the global power control subsystem during load-following transients. It should be stated that the power maneuvering is accomplished without reactivity compensation by a change in the boron concentration, which is one of the main benefits of the MSHIM control strategy over the traditional strategies.

The simulation results show that the proposed decoupling control system successfully can decouple the AP1000 reactor core system. In this manner, the AO can be controlled more tightly, and then the power change conditions, such as load-ramp, load-step, and load-following operations, can be performed flexibly and safely.

\section{Conclusions}

In this paper, a two-node discrete dynamic model of an AP1000 reactor core is established that is beneficial for controller design, especially to carry out studies on the control of the core axial power distribution. A digital PID decoupling control system based on QDRNN is designed to decouple the original reactor core control system of the AP1000. The decoupling analysis of the simulation results of the designed system is discussed. Compared with the original system, the results show that the designed decoupling control system can successfully decouple the AP1000 reactor core system. The control parameters of the global power control subsystem 
and the AO control subsystem can be tuned online by QDRNN, which means that the effects of unexpected perturbations can also be weakened by the self-regulation character of QDRNN. The load-following operation is also simulated to test the decoupling system. The results show that the designed decoupling control system can tighten the $\mathrm{AO}$ and increase the flexibility and safety of the load-following operation.

\section{Acknowledgements}

This work was supported by the National Science Foundation of China under Grants 11405125; China Postdoctoral Science Foundation Funded Project under Grant 2014M562420, and the Fundamental Research Funds for the Central Universities of China under Grant 2015gjhz09. 


\section{Nomenclature}

\begin{tabular}{|c|c|}
\hline$A O$ & axial offset \\
\hline$B$ & insertion position of control roads, $\mathrm{cm}$ \\
\hline$b_{1}, b_{2}, b_{3}$ & constant coefficients \\
\hline C & normalization of $c$ \\
\hline$c$ & delayed neutron precursor concentration, $\mathrm{m}^{-3}$ \\
\hline$c_{p}$ & specific heat, $\mathrm{J} \cdot \mathrm{kg}^{-1} \cdot \mathrm{K}^{-1}$ \\
\hline$D$ & diffusion coefficient \\
\hline$E$ & identifying index of QDRNN \\
\hline$F$ & coupling coefficient \\
\hline$f$ & fraction of the core power generated in the fuel \\
\hline$G$ & mass flow rate of coolant, $\mathrm{kg} \cdot \mathrm{s}^{-1}$ \\
\hline$I$ & input of QDRNN \\
\hline Io & normalization of $n_{I}$ \\
\hline$k$ & parameter of PID controller \\
\hline$L$ & height of the core, $\mathrm{m}$ \\
\hline$m$ & mass, $\mathrm{kg}$ \\
\hline & normalization of $n$ \\
\hline
\end{tabular}




\begin{tabular}{|c|c|}
\hline$n$ & neutron concentration, $\mathrm{m}^{-3}$ \\
\hline$n_{I}$ & iodine concentration, $\mathrm{m}^{-3}$ \\
\hline$n_{X}$ & xenon concentration, $\mathrm{m}^{-3}$ \\
\hline$O$ & output of QDRNN \\
\hline$P$ & power \\
\hline$S$ & recurrent of QDRNN \\
\hline$T$ & temperature, $\mathrm{K}$ \\
\hline$u$ & control input \\
\hline$v$ & neutron average speed, $\mathrm{m} \cdot \mathrm{s}^{-1}$ \\
\hline$W$ & connection weight matrices of QDRNN \\
\hline$X$ & output of recurrent layer of QDRNN \\
\hline$X e$ & normalization of $n_{X}$ \\
\hline Greek & \\
\hline$\alpha$ & reactivity coefficients \\
\hline$\beta$ & fraction of all delayed fission neutrons \\
\hline$\gamma$ & fission yield \\
\hline$\eta$ & learning rate \\
\hline
\end{tabular}




$\begin{array}{ll}\Lambda & \text { prompt neutron lifetime } \\ \lambda & \text { decay constant } \\ \mu & \text { heat capacity, } \mathrm{J} \cdot \mathrm{K}^{-1} \text {; learning rate } \\ \rho & \text { reactivity, pcm } \\ \Sigma_{f} & \text { macroscopic fission cross-section } \\ \sigma & \text { microscopic cross-section } \\ \tau & \text { sampling period }\end{array}$

\section{Subscripts}

0

steady-state

1

Node1

2

Node2

AO

AO; AO-bank

ave

average

$c$

coolant

$d$

differentiation parameter of PID

$f \quad$ fuel

$i \quad$ integration parameter of PID

in inlet 


$\begin{array}{ll}j & j \text { th delayed neutron group } \\ M & \text { M-banks } \\ \text { out } & \text { outlet } \\ P & \text { global power } \\ p & \text { proportional parameter of PID } \\ r & \\ & \text { reference value }\end{array}$

\section{Superscripts}

$\begin{array}{ll}D & \text { recurrent layer } \\ I & \text { input layer } \\ O & \text { output layer }\end{array}$




\section{References}

Dray, J. S., 1996. Dynamic recurrent neural networks: A dynamical analysis. IEEE Transaction on Systems, Man, and Cybernetics, Part B: Cybernetics. 26(5), 692-706.

Drudy, K. J., Mueller, N. P. Richter, E. A., 2013. Method of Achieving Automatic Axial Power Distribution Control. Pub. No.: US 2013/0101077A1., U.S. Patent Application Publication, 2013.

Drudy, K. J., Morita, T., Connelley, B. T., 2009. Robustness of the MSHIM operation and control strategy in the AP1000 design. ICONE17. Brussels, Belgium.

Franceschini, F., Petrovic, B., 2008. Advanced operational strategy for the IRIS reactor: Load follow through mechanical shim (MSHIM). Nuclear Engineering and Design. 238(12), 3240-3252.

Han, G., 2012. A PID Decoupling Controller Based on DRNN. International Journal of Advanced Computer Science. 2(5), 200-203.

Li, H., Wang, J., Gu, S., 2004. Study on a Quasi-Diagonal Recurrent Neural Network and Its Algorithm. Journal of Syetem Simulation. 16(7), 1542-1547 (in Chinese).

Li, H., Rong, L., Zhu, Y., 2007. Multivariable decoupling control of aeroengine based on QDRNN. Journal of Aerospace Power. 22(11), 1921-1924.

Liu, J., 2011. Advanced PID control MATLAB Simulation. Electronic Industry Press, Beijing.

Onoue, M., Kawanishi, T., Carlson, W. R., Morita, T., 2003. Application of MSHIM core control strategy for Westinghouse AP1000 cuclear power plant. GENES4/ANP2003. Kyoto, Japan.

Fetterman, R. J.. Advanced first core design for the westinghouse AP1000. ICONE17. July 12-16, 2009, Brussels, Belgium.

Schulz, T. L., 2006. Westinghouse AP1000 advanced passive plant. Nuclear Engineering and Design. 236(14), 1547-1557.

Wang, P., Wan, J., Yna, S., Liu, Y., Zhao, F., 2014 Application of an improved mechanical shim control strategy for AP1000 reactor. Nuclear Technology. 187, 243-259.

Wang, P., Liu, Y., Jiang, B., Wan, J., Zhao, F., 2013. Nodal dynamics modeling of AP1000 reactor for control system design and simulation. Annals of Nuclear Energy. 62(1), 208-223.

Westinghouse Electric Company, 2011. AP1000 Design Control Document, Rev. 19.

Westinghouse Electric Company, 2010. AP1000 Fuel Design \& Core Operations.

Zheng, X., Liu, Y., Sun, J., 2010. The peak load regulation of nuclear power plants in Guangdong power grid. Electric Power Technology. 19, 81-85 (in Chinese). 